\title{
Blocking time analysis of OBS routers with arbitrary burst size distribution
}

\author{
D. Morató, M. Izal, J. Aracil, E. Magaña, J. Miqueleiz \\ Dept. de Automática y Computación \\ Universidad Pública de Navarra, Pamplona, SPAIN \\ email:daniel.morato@unavarra.es
}

\begin{abstract}
The blocking time distribution for an OBS router is obtained, under the assumption of Poisson-arriving bursts with Pareto, Gaussian and Exponential burst size distributions. Analytical expressions are provided as a function of number of wavelengths per port. Such expressions can be used to dimension Fiber Delay Lines (FDLs) and to perform end-to-end delay estimation. On the other hand, we show that the blocking time distribution becomes exponential as the number of wavelengths increases, regardless of the burst size distribution. Since the burst size distribution is determined by the burst assembly algorithm at the network edges, we conclude that the burst assembly algorithm will have no influence on both burst blocking probability and burst blocking time in future DWDM networks.
\end{abstract}

Keywords: Optical Burst Switching, burst blocking time distribution.

\section{INTRODUCTION AND PROBLEM STATEMENT}

The advent of terabit optical networks requires the use of an efficient transfer mode for IP packets. In fact, switching IP packets, which have negligible transmission time, poses a number of challenges regarding synchronization and buffering in the all-optical routing elements. Alternatively, Optical Burst Switching (OBS) [1] provides "coarse packet switching" service in the optical network, namely a transfer mode which is halfway between circuit switching and pure packet switching. First, a control packet is sent along the path from source to destination, so that resources can be reserved for the incoming burst. Then, the data burst follows after an offset time. Among other features, OBS offers scope for differentiated quality of service, traffic engineering (GMPLS) and path protection and restoration [2].

Central to the OBS concept is the separation between data and control plane. Reservation messages are sent in separate wavelengths and suffer $\mathrm{O} / \mathrm{E} / \mathrm{O}$ conversion in the OBS router, since they are processed in the electronic domain. On the contrary, the burst is switched "cut-through" without leaving the optical domain. At any OBS router, a wavelength on the desired output link will be reserved using delayed reservation (DR) [1], i.e., the wavelength will be reserved from the burst arrival time to the burst departure time. However, if no wavelength is available, the incoming bursts will be blocked and a Fiber Delay Line (FDL) will be reserved using DR. If either no FDLs are available or the blocking time of the output port is larger than the delay time of the available FDLs then the burst will be dropped. Therefore, not only the burst dropping rate is influenced by the number of wavelengths, but also by their blocking time.

For bufferless OBS routers, the wavelength blocking probability can be estimated with the Erlang-B formula [3]-[6], under the assumption of Poisson-arriving bursts and arbitrary burst size distribution with finite first moment. In a previous paper [7], we show that the burst arrival process can be assumed to be Poisson, despite the possible long-range dependence of incoming traffic. On the other hand, the burst size distribution depends on the burst assembly algorithm which is used at the edges of the optical network, where the burst assembly process takes place. For example, the burst size turns out to be Gaussian for timer-based schemes [7]. Other non-Gaussian burst size distributions that have also been considered in the literature are the exponential distribution [3], [5], [6], the hyperexponential distribution [4], and the Pareto distribution [4]. Since they all have a finite first moment, the blocking probability is the same for all of them, as predicted by the $\mathrm{M} / \mathrm{G} / \mathrm{N} / \mathrm{N}$ analysis $[8$, section 5.5.2].

In this paper, the performance analysis of OBS routers is extended to the blocking time distribution. Assuming that FDLs are available in the OBS router, an incoming burst will be buffered if and only if its blocking time is smaller than the delay time provided by the FDL. Otherwise, the burst will be simply dropped. Therefore, knowledge of the blocking time is essential in order to determine the FDL length (delay time) for a given drop rate objective. It must be noted that even though a long maximum delay can reduce the blocking probability, one needs to keep it as short as possible [3]. Ideally, the delay incurred by a burst should be as close as possible to the blocking time, in order to reduce end-to-end delay and hardware size $(1 \mathrm{~km}$. FDLs at $2.5 \mathrm{Gbps}$ line speed are chosen in [9]). On the other hand, the blocking time has an influence on the total end-to-end delay for a burst. It is expected that time-constrained traffic, such as interactive video traffic, will be carried by the optical network. Furthermore, non-interactive services such as TCP services are also influenced by endto-end delay, since loss detection is timer-based. Eventually, expiration of ACK timers can make the TCP connection enter congestion avoidance, implying severe throughput degradation. In conclusion, knowledge of the blocking time distribution is a fundamental issue in the design of cost-effective OBS routers.

Our findings show that only the tail of the blocking time dis- 
tribution is affected by the burst size distribution. Furthermore, as the number of wavelengths per port increases, the blocking time distribution is approximately exponential. Due to the rapid optical technology development, the number of wavelengths per port is expected to increase in the close future. Thus, we conclude that, for DWDM networks, not only all burst assembly algorithms are equivalent in terms of blocking probability, but they are also equivalent in terms of blocking time distribution.

\section{A. Network scenario}

Figure 1 shows the scenario under analysis. Since incoming traffic to the OBS cloud comes in packets, burst assembly functionality is required at the edges. The edge nodes in charge of burst assembly will be called burstifiers. An OBS burstifier maintains separate queues per destination, namely separate queues per Forward Equivalence Class.

There is a choice of burst assembly algorithms depending on the type of traffic being transmitted. Timer-based algorithms are suitable for time-constrained traffic, since an upper bound to the burst assembly time is enforced. For non time-constrained bulk traffic, the objective is to improve transmission efficiency, despite of a larger burst assembly time. For example, a filesover-lightpaths transmission scheme for non-interactive TCP traffic is proposed in [10], that serves to reduce the control packets overhead and increase OBS transmission efficiency. Such scheme yields Pareto-distributed burst sizes. In conclusion, since different burst size distributions may arise from the different burst assembly algorithms we do not restrict our analysis to a single distribution, but, instead, Pareto, Gaussian and Exponential distributions are considered. On the other hand, the burst arrival process is assumed to be Poisson [3]-[7].

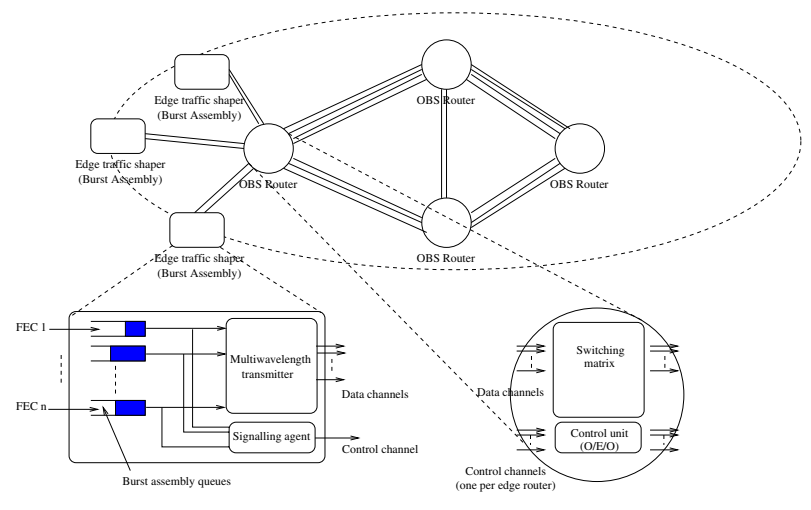

Fig. 1. Network reference model

The blocking time distribution for bursts arriving as a Poisson process is derived analitycally and by simulation. First, we provide an approximation for the blocking time distribution. For such approximation (exponential), it is only required that the burst size distribution has a finite first moment. Thus, the approximation applies to a broad range of possible burst size distributions. Additionally, the approximation is better the larger the number of wavelengths per port. This is consistent with the current technological trend in DWDM networking.
Second, exact analytical expressions for the blocking time distribution are obtained, for each of the three burst size distributions listed above. Such expressions are exact for any number of wavelengths.

The rest of the paper is organized as follows: in section II we define the analysis scenario and methodology, section III presents the analysis and section IV is devoted to results and discussion, followed by the conclusions that can be drawn from this paper.

\section{Methodology}

For simplicity, we focus on a given output port, where it is assumed that arrivals are Poisson. Furthermore, an incoming burst to the output port may be transmitted using any wavelength. Let $N$ be the number of wavelengths per output port, which will take on values 8 (CWDM) and 64 (DWDM). For example, a number of 32 wavelengths per fiber is reported for a recently developed photonic MPLS router [11].

On the other hand, the output port will be assumed to receive traffic from a large number of hosts which are sending data files over the optical network. Depending on the burst assembly algorithm the burst size distribution will be different. However, and in order to run simulations and numerical experiments under the same offered load, the average burst size will be the same regardless of the burst size distribution. Such average burst size, which will be denoted by $\bar{Z}$, is made equal to the average file size in the Internet (15 Kbytes), in accordance to recently reported measurements [12].

\section{A. Pareto distributed burst size}

Pareto-distributed burst sizes will arise if a single burst per file [10] is provided by the burstifier. In such case, the burst and file sizes are equal in distribution. Note that $Z$ is Paretodistributed iff

$$
\begin{aligned}
& P(Z>z)=1 \quad z \leq K \\
& P(Z>z)=K^{\alpha} z^{-\alpha} \quad z>K
\end{aligned}
$$

where $\alpha=1.4$ is the decay exponent and $K>0$ is the minimum burst size.

\section{B. Gaussian distributed burst size}

Timer-based burstifiers will give raise to (truncated) Gaussian-distributed burst sizes [7]. To fully characterize the distribution, the variance coefficient $C_{v}^{2}=\sigma^{2} / \bar{Z}^{2}$ is set to 0.2 .

\section{Exponentially distributed burst sizes}

The exponential distribution has been considered in [3], [4]. Such distribution is characterized by the average burst size (15 Kbytes).

Finally, the wavelength speed is set to $10 \mathrm{Gbps}$, and, thus, the average transmission time for a burst is equal to $12 \mu \mathrm{s}$. The same average transmission time is considered in [3]. 


\section{ANALYSIS}

In addition to the parameters provided in the previous section, let $R_{j}$ be the residual time in service (residual life) of the burst being transmitted in wavelength $j$, where $j=1, \ldots, N$, being $N$ the number of wavelengths per port (servers). The output port will be blocked if and only if $j=N$. Let $Y$ be the random variable that represents the blocking time for an incoming burst. We wish to derive $P(Y>y), y>0$, i.e. the survival function of the blocking time. Since burst arrivals are Poisson, and due to the PASTA property, the blocking time is given by the minimum of the residual lives of the $N$ bursts in service, namely $\{Y>y\}=\min _{j=1, \ldots, N}\left\{R_{j}>y\right\}$. Since bursts sizes are independent and identically distributed

$$
P(Y>y)=\prod_{j=1}^{N} P\left(R_{j}>y\right)=P\left(R_{1}>y\right)^{N}
$$

Equation (2) provides an expression for the blocking time distribution (survival function). Note that the blocking time distribution depends on the burst size distribution. First, under the weak assumption of finiteness in the burst size first moment, we will show that the blocking time survival function becomes exponential with the number of wavelengths $(N)$. Second, we will provide closed analytical expressions for (2) assuming Pareto, Gaussian and Exponential burst sizes.

\section{A. Approximations for the blocking time distribution}

The density of the residual life of a burst in service $\left(P\left(R_{1}>\right.\right.$ $y)$ ), as seen by Poisson arrivals, can be obtained from the survival function of the service time as follows [13, pp. 172, vol. I]

$$
\hat{f}_{R_{1}}(z)=\frac{P\left(X_{1}>z\right)}{E X_{1}}
$$

Thus,

$$
\begin{aligned}
& P\left(R_{1}>y\right)=\int_{y}^{\infty} \hat{f}_{R_{1}}(z) d z=\int_{0}^{\infty} \hat{f}_{R_{1}}(z) d z- \\
& -\int_{0}^{y} \hat{f}_{R_{1}}(z) d z=1-\frac{1}{E X_{1}} \int_{0}^{y} P\left(X_{1}>z\right) d z
\end{aligned}
$$

since $R_{1} \geq 0$ a. s. From the previous equation and (2)

$$
P(Y>y)=\left(1-\frac{1}{E X_{1}} \int_{0}^{y} P\left(X_{1}>z\right) d z\right)^{N}
$$

Since $P\left(X_{1}>z\right)$ is a monotonically decreasing function,

$$
\left(1-\frac{y}{E X_{1}}\right)^{N}<\left(1-\frac{1}{E X_{1}} \int_{0}^{y} P\left(X_{1}>z\right) d z\right)^{N}
$$

and

$$
\left(1-\frac{1}{E X_{1}} \int_{0}^{y} P\left(X_{1}>z\right) d z\right)^{N}<\left(1-\frac{y P\left(X_{1}>y\right)}{E X_{1}}\right)^{N}
$$

For values of $N$ and $y$ such that $N * P\left(X_{1}>y\right) \sim N$ (note that this happens for values of $y$ not in the tail of the distribution and for moderate to large number of wavelengths per port), (6) and (7) yield

$$
P(Y>y)=\left(1-\frac{1}{E X_{1}} \int_{0}^{y} P\left(X_{1}>z\right)\right)^{N} \approx e^{-\frac{N y}{E X_{1}}}
$$

In order to derive the last equation, it has been taken into account that $e^{-x}=1-x+o(x)$. This approximation is more accurate the smaller the value of $y$ and the larger the value of $N$. The numerical results in the next section will serve to demonstrate the accuracy of this approximation.

\section{B. Pareto-distributed burst sizes}

An exact expression for the blocking time distribution can be found using (2). Substitution of (1) in (4) yields

$$
\begin{array}{lr}
P\left(R_{1}>y\right)=\frac{(\alpha-1)(K-y)+K}{\alpha K} & 0 \leq y \leq K \\
P\left(R_{1}>y\right)=\frac{K^{(\alpha-1)}}{\alpha} y^{(1-\alpha)} & y>K
\end{array}
$$

In order to derive the last equation, it must be noted that $E X_{1}=\alpha K /(\alpha-1)$. Thus, using (2),

$$
\begin{array}{lr}
P(Y>y)=\left(\frac{(\alpha-1)(K-y)+K}{\alpha K}\right)^{N} 0 \leq y \leq K \\
P(Y>y)=\left(\frac{K^{(\alpha-1)}}{\alpha} y^{(1-\alpha)}\right)^{N} \quad y>K
\end{array}
$$

\section{Gaussian-distributed burst sizes}

The same procedure can be adopted for the Gaussian case. First, the survival function of the service time is equal to

$P\left(X_{1}>z\right)=\phi\left(\frac{z-E X_{1}}{\sigma}\right)=\int_{z}^{+\infty} \frac{1}{\sqrt{2 \pi} \sigma} e^{\frac{-\left(x-E X_{1}\right)^{2}}{2 \sigma^{2}}} d x$

where $\phi(x)$ is the survival function $P(X>x)$ of the standard Gaussian random variable ${ }^{1}$. Finally, using (4) and (2),

$$
\begin{aligned}
& P(Y>y)= \\
& =\left(\frac{\sigma}{\sqrt{2 \pi} E X_{1}} e^{\frac{-\left(y-E X_{1}\right)^{2}}{2 \sigma^{2}}}+\frac{E X_{1}-y}{E X_{1}} \phi\left(\frac{y-E X_{1}}{\sigma}\right)\right)^{N}
\end{aligned}
$$

The proof is given in the appendix.

\footnotetext{
${ }^{1}$ Truncation to the positive values is assumed
} 


\section{Exponentially-distributed burst sizes}

The exponential case is straightforward due to the memoryless property of the exponential distribution, which implies that

$$
P\left(R_{1}>y\right)=e^{-\frac{y}{E X_{1}}}
$$

and, thus, we use (2) to obtain

$$
P(Y>y)=e^{-\frac{N y}{E X_{1}}}
$$

which is equal to the approximation (8).

\section{RESULTS AND DISCUSSION}

Numerical results from the above expressions are presented in this section, together with simulation results. In order to verify the accuracy of the approximation in section III-A both small and large values of $N$ (8 and 64) were selected. On the other hand, a trace-driven analysis was also conducted. Figure 2 shows the blocking time distribution (survival function) for $N=8$ and Pareto, Gaussian and Exponential burst size distributions. The traffic intensity is 4.5 Erlangs and the blocking probability is equal to 0.0483 . We observe that the approximation in section III-A is accurate for the lower part of the distribution, while the behavior of the distribution tail is influenced by the burst size distribution. Nevertheless, it must be noted that the expressions (10), (12) and (14) follow closely the simulation results in all the range of blocking time values.
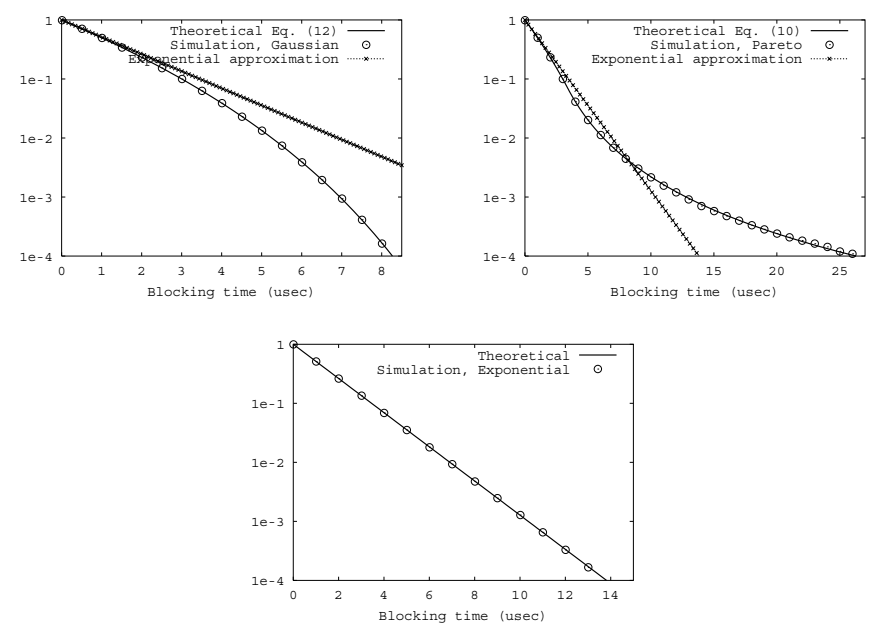

Fig. 2. Blocking time distribution (Gaussian -top left-, Pareto -top right-, Exponential -bottom-) $(N=8)$

On the other hand, figure 3 shows the blocking time distribution (survival function) for $N=64$. In order for the blocking probability to remain the same $(0.0483)$, the traffic intensity is now increased to 58.6 Erlangs. In this case, we observe that the exponential approximation is accurate on a larger part of the distribution. Both numerical results and simulations for values of $N$ beyond 64 show that the larger the value of $N$ the better the approximation. Note that this is consistent with the technological trend in DWDM networks, that will incorporate a large number of wavelengths per fiber in the close future.
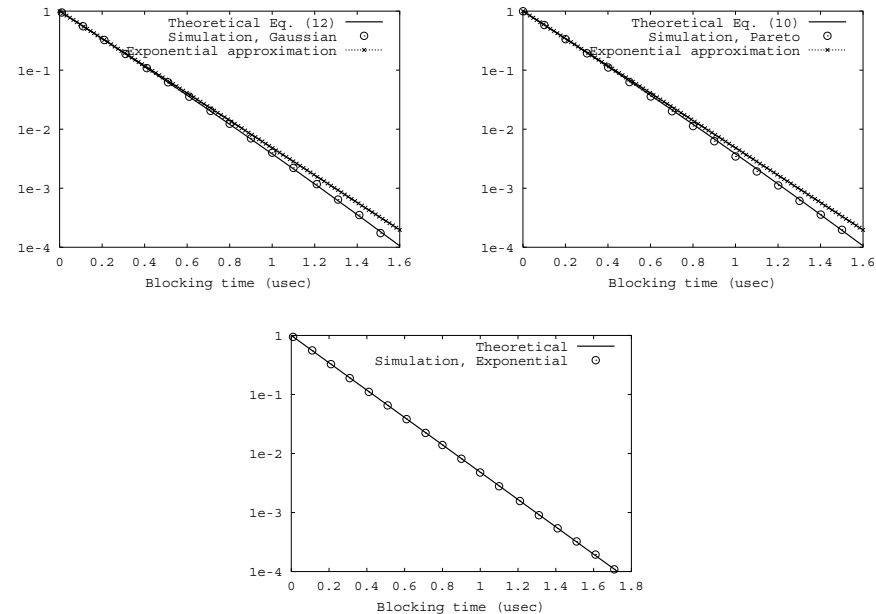

Fig. 3. Blocking time distribution (Gaussian -top left-, Pareto -top right-, Exponential -bottom- $)(N=64)$

\section{A. Trace-driven simulation}

A trace-driven simulation was also conducted in order to further assess that the analytical results presented in this paper apply to a real Internet scenario. The number of wavelengths per port $(N)$ is also made equal to 8 and 64 , as in the previous section. The trace was obtained from the NLANR Passive Measurement and Analysis research project ${ }^{2}$ (Abilene-I data set). A two hours trace from two bidirectional OC48 links was used for the simulations. These links connect the Internet 2 Indianapolis node with the Cleveland node and the Kansas City node. A timer-based burst assembly technique was adopted, with a $300 \mu$ s. timer. Incoming packets to the edge shaper are demultiplexed according to their destination in separate queues. A timer is started with the first packet in the queue. Upon timeout expiry, the burst is assembled and relayed to the transmission queue. For this particular burstifier, we have shown that the burst size distribution turns out to be (truncated) Gaussian [7]. Being the burst size distribution Gaussian, we expect that the blocking time distribution is well modeled by (12). On the other hand, the approximation (8) is also expected to apply, specially as the number of wavelengths increases. Figure 4 shows the blocking time distribution for $N=\{8,64\}$.
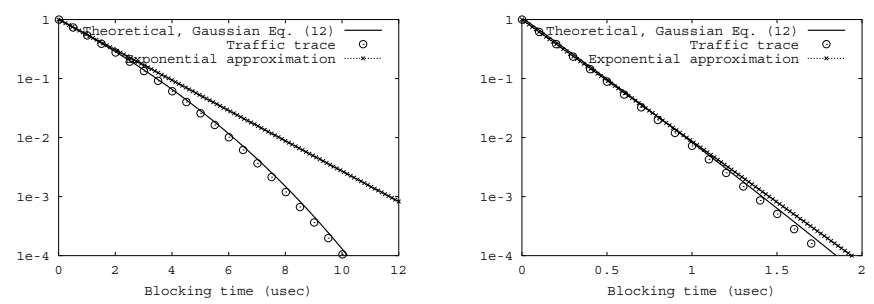

Fig. 4. Blocking time distribution (Trace-driven simulation $N=8$ (left), $N=64$ (right))

The results show very good agreement between the exact

${ }^{2}$ http://pma.nlanr.net 
analytical distribution (12) and the empirical blocking time distribution. On the other hand, the accuracy of the approximation (8) increases with the number of wavelengths, and it is a good approximation for $N=64$. In conclusion, simulations with both real traffic and synthetic traffic clearly show that our analysis can be applied to a wide range of OBS scenarios.

\section{Conclusions}

Optical Burst Switching is a promising transport technique for the optical backbone. However, burst assembly functionality is required at the network edges and a number of algorithms can be used for this purpose, that will surely give raise to different burst size distributions. In this paper we address the fundamental issue of how the OBS routers are affected by the burst assembly algorithm. The state of the art [3]-[6] shows that the burst blocking probability does not depend on the burst size distribution, namely, blocking probability does not depend on the burst assembly algorithm. Under the weak assumption of finiteness in the burst size first moment, we show that the blocking time distribution becomes independent from the burst assembly algorithm as the number of wavelengths per fiber increases. Thus, an exponential approximation for the blocking time distribution is presented, for the case of moderate to large number of wavelengths (64). Additionally, exact expressions for the blocking time distribution are provided, for any number of wavelengths, considering three common burst size distributions (Pareto, Gaussian, Exponential). These results apply directly to FDL dimensioning and end-to-end delay estimation.

\section{ACKNOWLEDGMENTS}

The authors are grateful to the National Science Foundation (cooperative agreement ANI-9807479) and to the NLANR Measurement and Network Analysis group for providing access to the traffic traces used in this paper.

\section{APPENDIX}

\section{PROOF OF (12)}

Since $X_{1}$ is Gaussian with mean $E X_{1}$ and variance $\sigma^{2}$ then,

$P\left(X_{1}>z\right)=\phi\left(\frac{z-E X_{1}}{\sigma}\right)=\int_{z}^{+\infty} \frac{1}{\sqrt{2 \pi} \sigma} e^{\frac{-\left(x-E X_{1}\right)^{2}}{2 \sigma^{2}}} d x$

and, using (4),

$$
P\left(R_{1}>y\right)=\frac{1}{E X_{1}} \int_{y}^{+\infty} \int_{z}^{+\infty} \frac{1}{\sqrt{2 \pi} \sigma} e^{\frac{-\left(x-E X_{1}\right)^{2}}{2 \sigma^{2}}} d x d z
$$

By Fubini's Theorem,

$P\left(R_{1}>y\right)=\frac{1}{E X_{1}} \int_{y}^{+\infty} \int_{y}^{x} \frac{1}{\sqrt{2 \pi} \sigma} e^{\frac{-\left(x-E X_{1}\right)^{2}}{2 \sigma^{2}}} d z d x$

and, thus,

$$
\begin{aligned}
P\left(R_{1}>y\right) & =\frac{1}{E X_{1}} \int_{y}^{+\infty} x \frac{1}{\sqrt{2 \pi} \sigma} e^{\frac{-\left(x-E X_{1}\right)^{2}}{2 \sigma^{2}}} d x \\
& -\frac{1}{E X_{1}} \int_{y}^{+\infty} y \frac{1}{\sqrt{2 \pi} \sigma} e^{\frac{-\left(x-E X_{1}\right)^{2}}{2 \sigma^{2}}} d x
\end{aligned}
$$

Now, solve the previous integral to obtain

$$
\begin{aligned}
P\left(R_{1}>y\right) & =\frac{\sigma}{\sqrt{2 \pi} E X_{1}} e^{\frac{-\left(y-E X_{1}\right)^{2}}{2 \sigma^{2}}}+ \\
& +\frac{E X_{1}-y}{E X_{1}} \phi\left(\frac{y-E X_{1}}{\sigma}\right)
\end{aligned}
$$

which, together with (2) yields (12).

\section{REFERENCES}

[1] C. Qiao and M. Yoo, "Optical burst switching (OBS) - A new paradigm for an optical Internet," Journal of High-Speed Networks, vol. 8, no. 1, 1999.

[2] C. Qiao, "Labeled optical burst switching for IP-over-WDM integration," IEEE Communications Magazine, vol. 38, no. 9, pp. 104-114, 2000.

[3] M. Yoo, C. Qiao, and S. Dixit, "QoS performance of optical burst switching in IP over WDM networks," IEEE Journal of Selected Areas in Communications, vol. 18, no. 10, pp. 2062-2071, October 2000.

[4] K. Dolzer, C. Gauger, J. Spath, and S. Bodamer, "Evaluation of reservation mechanisms for optical burst switching," International Journal of Electronics and Communications (AE), vol. 55, no. 1, 2001.

[5] S. Verma, H. Chaskar, and R. Ravikhant, "Optical burst switching: A viable solution of terabit IP backbone," IEEE Network, November/December 2000.

[6] M. Yoo and C. Qiao, "Supporting multiple classes of services in IP over WDM networks," in Proceedings of GLOBECOM 1999, Rio de Janeiro, Brazil, 1999.

[7] M. Izal and J. Aracil, "On the influence of self-similarity in optical burst switching traffic," in Proceedings of GLOBECOM 2002, Taipei, Taiwan, 2002.

[8] D. Gross and C. M. Harris, Fundamentals of queueing theory. John Wiley and Sons, 1998.

[9] S. Yao, F. Xue, B. Mukherjee, S. Yoo, and S. Dixit, "Electrical ingress buffering and traffic aggregation for optical packet switching and their effect on TCP-level performance optical mesh networks," IEEE Communications Magazine, vol. 40, no. 9, pp. 66-72, September 2002.

[10] M. Izal and J. Aracil, "IP over WDM dynamic link layer: challenges, open issues and comparison of files-over-lighpaths versus photonic packet switching," in Proceedings of SPIE OptiComm 2001: Optical Networking and Communications, Denver, USA, August 2001.

[11] S. Okamoto, E. Oki, A. Sahara, and N. Yamanaka, "Demostration of the highly reliable HIKARI router network based on a newly developed disjoint path selection scheme," IEEE Communications Magazine, vol. 40, no. 11, pp. 52-59, November 2002.

[12] A. Downey, "Evidence for long-tailed distributions in the Internet," in Proceedings of ACM SIGCOMM Internet Measurement Workshop, 2001.

[13] L. Kleinrock, Queueing Systems. John Wiley and Sons, 1975. 